\title{
Left Hemicolectomy for Intussusception of the Transverse Colon Caused by a Giant Benign Lipoma
}

\author{
Author: \\ Kevin P. Birmingham \\ General Surgery Department, Daisy Hill Hospital, Southern Healthcare Trust, Northern \\ Ireland, UK \\ Correspondence to kevin.birmingham@nhs.net \\ Disclosure: \\ The author has declared no conflicts of interest. \\ Received: \\ 05.12 .20 \\ Accepted: \\ 03.02.21 \\ Keywords: \\ Colocolonic, diverticulitis, intussusception, lipoma, polyps. \\ Citation: \\ EMJ Radiol. 2021;2[1]:90-93.
}

\begin{abstract}
Colocolonic intussusception, caused by submucosal lipomas, is extremely rare. These benign soft tissue tumours comprise mature adipocytes of mesenchymal origin. While the majority of patients with lipomas remain asymptomatic, large or giant size lipomas $(>4 \mathrm{~cm}$ ) have been shown to cause debilitating abdominal pain, alternating bowel pattern, and anaemia secondary to gastrointestinal blood loss. This necessitates intervention in the form of surgical resection or endoscopic removal. However, once lipomas increase beyond $2 \mathrm{~cm}$ in size there is a significant risk of complications with an endoscopic approach, and open surgery or laparoscopic resection with bowel re-anastomosis is warranted. In this case put forth, the patient underwent a successful transverse colectomy and primary anastomosis.
\end{abstract}

\section{INTRODUCTION}

\section{CASE REPORT}

Colonic lipomas are nonepithelial benign submucosal tumours, most commonly presenting in adults in their 50 s and 60s. ${ }^{1}$ CT is the gold standard imaging modality and is often used first-line given the clinical presentation and lipomas propensity to mimic colorectal carcinoma. Barium studies are nondiagnostic and require biopsy and histopathological staining for definitive diagnosis. Most often, patients with lipomas remain asymptomatic until the lipoma exceeds $4 \mathrm{~cm}$ in size, at which stage they can cause intermittent obstruction. ${ }^{2}$

A 47-year-old female with an established background of diverticulosis presented with left iliac fossa pain and haematochesia associated with nausea and bloating. This was her third admission to hospital in 3 weeks. Her previous stays were short and her symptoms were presumed to be attributed to recurrent episodes of diverticulitis. On each occasion these symptoms had settled relatively quickly with intravenous amoxicillin and metronidazole. She had subsequently been discharged and scheduled for an outpatient sigmoidoscopy.

Her past surgical and medical history included open appendicectomy, endometrial ablation, hypertension, and acne vulgaris. Notably, there were several inpatient stays for diverticulitis 
during the preceding years, and imaging had shown extensive diverticular disease of the sigmoid colon with inflammatory changes and mucosal thickening of the rectum and sigmoid. During one of these inpatient admissions 2 years prior, an incidental lipoma measuring $4.7 \mathrm{~cm}$ was seen at the distal transverse colon on CT imaging. Simultaneously, a polyp of about $2.5 \mathrm{~cm}$ in size and pedunculated at the rectus sigmoid junction was removed at colonoscopy; the helix pomatia agglutinin results revealed this to be a hypoplastic polyp.

Her blood panel on current admission showed the following: haemoglobin $110 \mathrm{~g} / \mathrm{L}$, mean cell volume $96 \mathrm{fL}$, white cell count $7.2 \times 10^{9} / \mathrm{L}$, platelets $339 \times 10^{9} / \mathrm{L}, \quad$ C-reactive protein 31 $\mathrm{mg} / \mathrm{L}$, and $\mathrm{V}$-glutamyl transferase $73 \mathrm{lU} / \mathrm{mL}$. CT imaging of the abdomen and pelvis revealed an intussusception of the distal transverse colon into the descending colon. This intussusception was approximately $10 \mathrm{~cm}$ long, with the lead point being a large intramural lipoma measuring $6 \mathrm{~cm}$ in diameter (Figure 1 and 2). The proximal colon was normal in calibre and nondistended with no obvious small bowel obstruction evident. There was no fluid collection or free gas. Imaging was otherwise typical apart from multiple hepatic hypodensities which were consistent with cysts.

The patient underwent a left hemicolectomy and approximately $22 \mathrm{~cm}$ of colon with attached omentum was resected. This procedure best suited a laparoscopic approach but because the patient presented during the peak response of the coronavirus disease (COVID-19), local hospital policies precluded an aerosol generated procedure; therefore, it was carried out as open surgical procedure with full personal protective equipment precautions.

There were no intraoperative complications encountered and the patient made a swift postoperative recovery, apart from a sequential drop in haemoglobin to $54 \mathrm{~g} / \mathrm{L}$ on the postoperative day, which required a transfusion of three units of packed red blood cells. A chest X-ray at this point showed normal pulmonary vasculature with no focal consolidation but mild right basal atelectasis. The patient was discharged home on postoperative Day 7.

The final pathology report described a firm bulge within the lumen measuring $55 \mathrm{~mm} \times 40$ $\mathrm{mm} \times 40 \mathrm{~mm}$, which was consistent with a large polyp; it had a smooth surface and was yellow at the tip, yet was more mottled and darker towards the base. The polyp was the locus of the intussusception. The mucosa around the base of the polyp was roughened and irregular. On sectioning, the polyp comprised solid fatty material with no haemorrhagic or cystic areas seen.

Histology of the sections from the polyp showed mature adipose tissue divided into lobules by fine fibrovascular septae. There were areas of necrosis within it and characteristics of a lipoma with no features to suggest malignancy. Histology of the mucosa overlying and surrounding the lipoma displayed focal regions of ulceration. Within these locations the mucosa also showed features of an ischaemic pattern of injury consistent with changes seen in intussusception with no dysplasia or malignancy. There were areas of fibrosis and increased vascularity within the serosa. Longitudinal resection margin showed a mild purulent exudate on the serosa that was otherwise typical. Histological analysis of sampled lymph nodes showed no primary or secondary tumour. The overall impression of the histological report was intussusception of the large colon around a lipoma within its wall.

\section{DISCUSSION}

The most common locations for colonic lipomas are the ascending colon (45.0\%), sigmoid colon (30.3\%), descending colon (15.2\%), and with the transverse colon being the least common (9.1\%). Almost $90 \%$ of colonic lipomas are localised to the submucosa with the rest confined to the subserosal or intramucosal layer. ${ }^{3}$

Secondary intussusception is defined as a pathological lesion of the intestinal wall that alters normal peristaltic activity and serves as a lead point, as in this instance (in the absence of a lead point it is classified as primary or idiopathic). Approximately $60-65 \%$ of intussusceptions in the large intestine have a malignant aetiology, making colonic lipomas much less common. ${ }^{4}$ Clinically, they can present insidiously with nonspecific signs and symptoms, which can persist for years and lead to misdiagnoses unless otherwise meticulously investigated. 


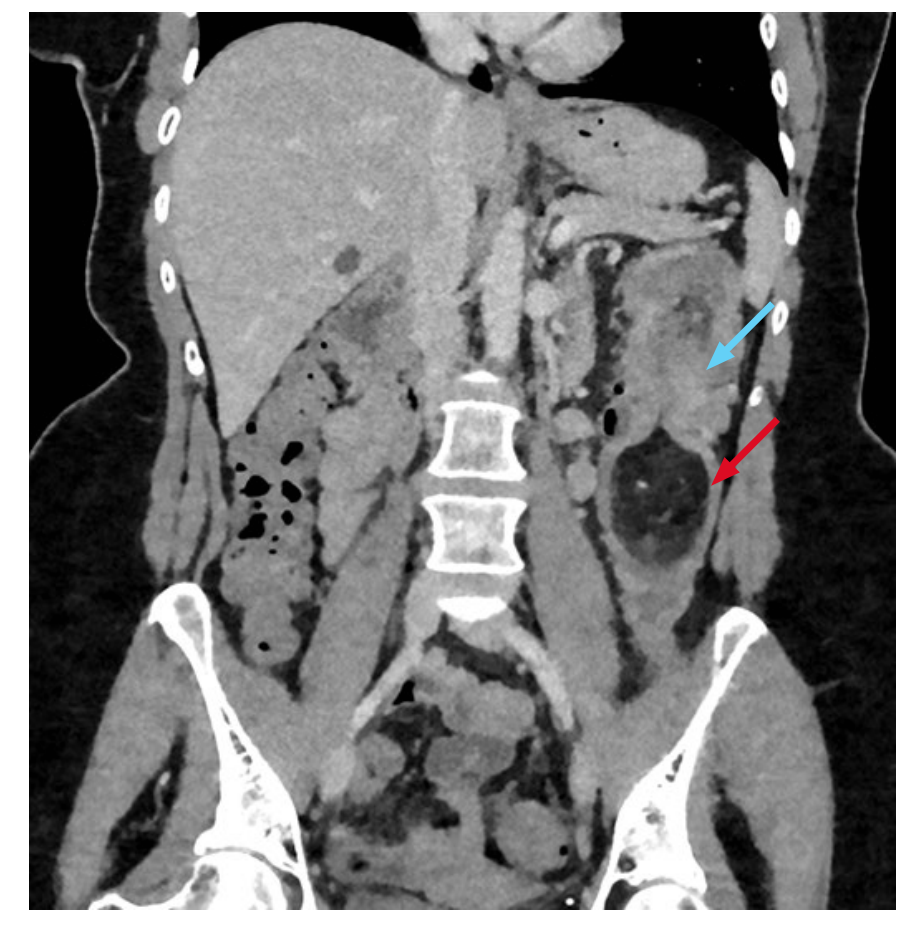

Figure 1: Coronal section of contrast enhanced abdominal CT showing intussusception (blue arrow) into the descending colon with lipoma as lead point (red arrow).

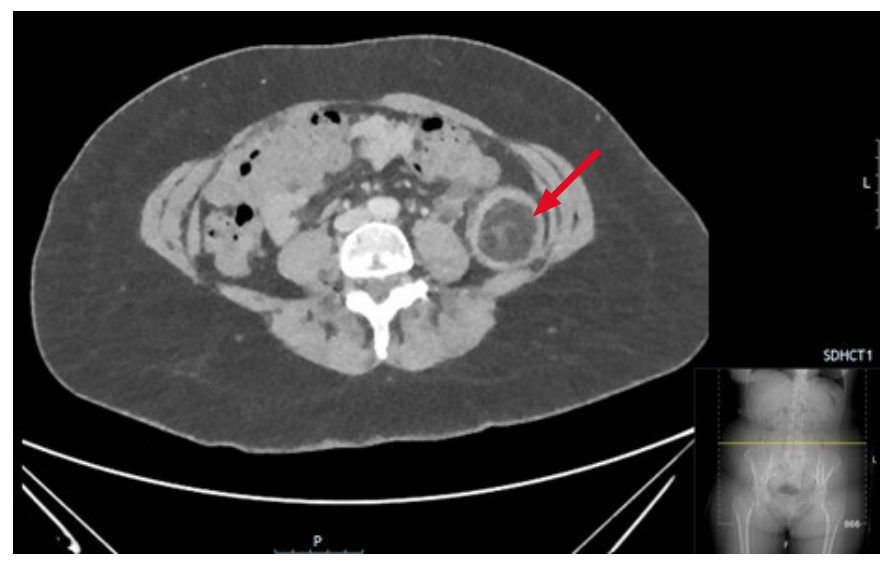

Figure 2: Axial view of soft tissue lipoma (arrow) filling the luminal space of the descending colon.

It is clear that fast growing colonic lipomas causing obstructive symptoms need to be removed. Ideally, lipomas $<2 \mathrm{~cm}$ in diameter can be removed endoscopically by snare and laser techniques. ${ }^{5}$ There are well-documented pathognomonic signs on endoscopic evaluation, such as the 'tenting' sign (mucosa grabbed over the lesion leaves a tented appearance) and the 'naked fat' sign (fat grossly extruded after biopsy). ${ }^{6}$
Giant lipomas (>4 cm in diameter) are more susceptible to complications such as superficial ulceration, haemorrhage, and obstruction. They can also undergo intermittent torsion and ischaemia. Therefore, these sizeable tissue masses require careful resection within the contained bowel segment and assiduous haemostasis with primary anastomosis, instead of submucosal lift techniques. Sessile polyps can prove difficult to ensnare. In addition, the low water content of fat can lead to prolonged 
electrocautery and risk of thermal damage. There is also a chance that the muscularis propria or serosal layer is involved. If so, these layers may become invaginated in the pedicle and increase the risk of perforation if endoscopic snare resection is used. ${ }^{7}$ For these reasons segmental surgical resection is safer and has the added benefit of allowing local lymph node dissection if the diagnosis of colonic carcinoma is equally equivocal.

\section{CONCLUSION}

Invariably, due to the nonspecific presentation of colonic lipomas, diagnosis is difficult and can be delayed. From the outset, symptoms of a large colonic lipoma are viewed adversely as they can mimic malignancy. Giant lipomas $(>4 \mathrm{~cm}$ ) can be technically challenging to remove endoscopically with an associated increased safety risk to the patient. In order to avoid complications these giant sized lipomas are managed most appropriately by surgical resection.

\section{References}

1. Michowitz $\mathrm{M}$ et al. Lipoma of the colon. A report of 22 cases. Am Surg. 1985;51(8):449-54.

2. Bahadursingh AM et al. Giant submucosal sigmoid colon lipoma. Am J Surg. 2003;186:81-2.

3. Rogy MA et al. Submucous largebowel lipomas - presentation and management. An 18-year study. Eur J
Surg. 1991;157:51-5.

4. Mohamed M et al. Adult sigmoidorectal intussusception related to colonic lipoma: a rare case report with an atypical presentation. Int J Surg Case Rep. 2015;10:134-7.

5. Mason $\mathrm{R}$ et al. Education and imaging. Gastrointestinal: lipoma induced intussusception of the transverse colon. J Gastroenterol Hepatol. 2010;25:1177.

6. De Beer RA, Shinya H. Colonic lipomas. An endoscopic analysis. Gastrointest Endosc. 1975;22:90-1.

7. Kim CY et al. Endoscopic removal of large colonic lipomas. Gastrointest Endosc. 2002;55:929-31. 